\title{
Faster Recovery after Exercise with Phytochemicals aimed at Mitochondrial Energy Turnover \\ - A double blind randomized placebo control study in college female soccer players -
}

\author{
E. Freye, MD, PhD \\ HP Strobel, RPharmD \\ From the Scientific Department, LaboGalenics Inc \\ Davos-Platz/Switzerland \\ Address for correspondence: E. Freye, MD, PhD \\ LaboGalenics Inc., Scientific Department, \\ Färbistr 3, 7270 Davos-Platz, Switzerland \\ e-mail: enno.freye@gmail.com \\ Phone: 0041-77-460-6962
} Keywords: ATP synthesis, mitochondria, Ubiquinol $(\mathrm{Q} 10-\mathrm{H} 2), \mathrm{NADH}(\mathrm{Q} 1)$, Siberian ginseng,
eleutherosides B \& E, ribose, heart rate recovery rate

\begin{abstract}
In order to replenish energy stores after exercise we developed a nutraceutical containing ingredients that precisely fuel the ATP generating mitochondria thus resulting in a faster recovery after workout.

To test the working hypothesis 18 healthy female college soccer players (mean age 18) enrolled in a short but extreme workout on the treadmill for $12 \mathrm{~min}$ as demonstrated in their rise in lactic acid. The volunteers were given an energy drink containing Q10-H2 (ubiquinol), NADH (nicotinamideadenine-dinucleotide-hydride or Q1), ribose, eleutherosides B \& E, Vit B2 \& B3, passion flower and Vit E over a period of one week. Following a washout of 4 weeks a similar looking and tasting placebo solution was given in a double blind randomized crossover design. Heart rate recovery rate and systolic blood pressure demonstrated a significant faster return to base line than after placebo within the first 1-5 minutes following exercise. In addition, ATP regeneration capacity in leucocytes following washout of sodiumazide, a specific inhibitor at the mitochondrial electron transport complex V, was statistically higher $(\mathrm{p}<0.05)$ in subjects after verum.

The composition consisting of mitochondrial active substances, results in a higher synthesis rate of ATP, which coincided with a faster heart rate recovery rate after workout.
\end{abstract}

\section{Introduction}

After an intensive workout, muscles have expended their available energy stores and need carbohydrates and protein in order to replenish and rebuild energy stores. Even if one is walking, and especially after an intense session of endurance running, there is the need for a recovery time to refill energy stores and it is commonly accepted that one gram of carbohydrates per kilogram of body weight per hour is needed for recovery, while adding protein in a 1:4 ratio, allowing the body to use the carbohydrates more efficiently to rebuild energy stores in the muscles. Because post exercise recovery is mandatory, nutritional intake is important to replenish endogenous substrate stores and to facilitate muscle-damage repair and reconditioning. After exhaustive endurance-type exercise, muscle glycogen repletion purportedly forms the most important factor determining the time needed to recover. It does not take into account how this type of energy is transformed into adenosine triphosphate (ATP), the necessary form of energy within the cell that keeps the muscle working. And although, consuming carbohydrate and protein during the early phases of recovery has been shown to positively affect subsequent exercise performance (Pedersen, Lessard et al. 2008), it does not address the importance of mitochondria within the muscle cells, which are responsible for a sufficient ATP synthesis and for the recovery of muscle strength. We therefore set 
out designing a new power drink, which composed those necessary supplements, considered mandatory to set the mitochondrial energy chain into action thus restoring energy, which is necessary for fast regeneration. In order to underline the efficacy and practical advantages of such newly created power drink we used the speed at which the heart rate recovers following a period of max endurance workout on the treadmill, while at the same time determining in vitro ATP regeneration capacity in leucocytes of tested subjects.

\section{Material and methods}

After having received informed consent from all participants and also having received approval from the local college committee that experiments are in compliance of the law as well as having received consent from the Swiss soccer union that the study was within their guidelines of ethics and did not involve any kind of doping, 18 healthy young female college soccer players (table 2) underwent a max workout on the treadmill on two separate occasions with 4 weeks apart. Prior to

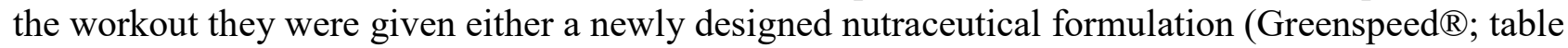
1), or a similar looking and tasting placebo solution for 1 week prior to exercise. For max workout they were exposed to a treadmill exercise reaching max heart rates $>180$ beats/min after 12 minutes.

For continuous recording of heart rate subjects were attached to a heart rate monitor (Polar® FT 40 heart rate monitor, Polar Electro $\mathrm{GmbH}$, Switzerland), which picked up and transmitted heart rate on-line during and especially in the post work-out period via a coded chest transmitter imbedded in an elastic electrode strap (Polar T31) for transmitting heart rate to the wrist unit. From this wrist unit data was later derived by use of a conventional computer depicting individual max heat rate decline at the end of the workout session and in the immediate post exercise period at 1-, 2- and 5 minutes intervals. This index in conjunction with the blood pressure measurements were used as the ability of the cardiovascular system to achieve a sufficient restoration of energy within the working musculature and especially the heart muscle when compared to control (Dimkpa 2009). Aside from heart rate, the blood pressure (systolic and diastolic) was determined from the pulse pressure curve using a medical blood pressure wrist measurement system (Bosco ${ }^{\circledR}$ company, Zürich/Switzerland) at similar time intervals as heart rate.

Aside from cardiovascular parameters, and following puncture of the index finger tip with a hemostat, blood sugar and lactate level were determined in all subjects before and after the exercise using a conventional hand held blood sugar/lactate monitoring system with the aid of calibrated glucose/lactate strips. Also, since ATP regenerating capacity is a rate-limiting step before hydrolysis drops below the critical $55 \mathrm{~kJ} / \mathrm{mol}$, the importance of local ATP regeneration capacity is of relevance as it increases in parallel with an increase in the rate of ATPase turnover (Korge 1995). We therefore set out to determine the individual ATP regeneration capacity, which has a similar relevance as the mitochondrial activity, being generated at complex $\mathrm{V}$ of the mitochondrial chain. In short, leucocytes of all subjects were exposed in-vitro to sodium azide a specific ATPase inhibitor (Bowler, Montgomery et al. 2006), resulting in a specific but reversible inhibition of the mitochondrial electron transport chain at complex $\mathrm{V}$ as described in detail elsewhere (KamMing and Hoi 2007) (Ko, Leon et al. 2006). At the end of the intake of either placebo or verum and prior to endurance running, basal ATP in leucocytes of participants was determined. This was followed by an induced inhibition of ATP synthesis with sodium azide. After washout it resulted in regeneration of the electron transport chain with a recovery in ATP concentration. With normative values $>500 \mathrm{pmol} / 10^{6}$ cells the recovery ATP should exceed the inhibitory concentration by at least $25 \%$ in order to demonstrate a sufficient mitochondrial recovery capacity (Biovis Laboratory Company, Limburg/Germany).

The verum (energy drink) contained the following ingredients adjusted with water to a vial of $50 \mathrm{ml}$ (table 1), while the placebo consisted of a pure fruit juice drink ("Take it Easy Michel®" Rivella company/Switzerland) of similar color and taste. All volunteers had to undergo two sessions of max workout on the treadmill and without prior knowledge, either a similar looking and tasting placebo drink or the energy drink was taken for one week, as generated by the computer. 


\section{Statistics}

All statistical analysis was performed with the Prism 5 software for Mac OS X (Graph Pad Software Inc. San Diego, USA). The normally distributed data were compared using student-t test. For comparison of skewed data Mann Whitney-U test was applied. Group differences were computed for statistical difference using the Newman Keuls multiple comparison test or the paired t-test when indicated, whichever was applicable. All statistical tests were two-sided and were considered as significant at the $\mathrm{p}<0.05$ level.

Sample size was calculated presuming a $70 \%$ incidence of difference in heart rate recovery in volunteers after treadmill exercise with and without the nutraceutical drink. Power analysis assumed an at least $30 \%$ faster decline in heart rate after exercise following consumption of the energy drink. With a value of $\alpha=0.05$ and $\beta=0.90$ it was computed that at least 12 subjects were required in each group. To minimize the effect of data loss a total of 18 volunteers were enrolled.

\section{Results}

Following a forced treadmill exercise, subjects in the placebo and in the verum group had a similar running distance with both groups reaching a max heart rate above a mean of 180 beats $/ \mathrm{min}$ at the end of endurance running, which was statistically not significant (table 2). Max workout was also reflected in a rise of lactate production, which was not significant between both groups (table 2).

However, within the recovery period there was a significant faster decline in heart rate in the verum compared to the placebo group in the $1 \mathrm{st}(\mathrm{p}<0.005)$ and in the 5 th $\min (\mathrm{p}<0.05)$ post exercise (fig. $1)$.

In addition to the difference in heart rate recovery between the two groups, a similar difference is also reflected in the decline of post exercise elevated systolic blood pressure (fig. 2). Although mean systolic pressure immediately following the exercise period was lower in the placebo when compared to the verum group, this difference is statistically not different because of the large standard deviation in the placebo group (fig. 2).

Compared to placebo there is a faster decline in elevated mean systolic blood pressure in the verum group. This decline is statistically significant $(\mathrm{p}<0.05)$ in the verum group at the 5 th minute after workout, illustrating a faster recovery.

Compared to systolic pressure there was no difference in the recovery rate in the diastolic blood pressure. Compared to the resting diastolic pressure both groups of subjects demonstrated values of $80 \mathrm{mmHg}( \pm 9.7 \mathrm{SD})$ and $80( \pm 11 \mathrm{SD})$ respectively in the 1 st post exercise minute. Thereafter diastolic pressure dropped to $68 \mathrm{mmHg}( \pm 9 \mathrm{SD})$ and $70 \mathrm{mmHg}( \pm 11 \mathrm{SD})$ in the verum and the placebo group respectively within the 5 th minute post exercise, which statistically is not significant. Aside from cardiovascular parameters, blood chemistry changes within the blood sugar levels indicate a highly significant $(\mathrm{p}<0.005)$ increase in the immediate post exercise period in the verum compared to the placebo group (table 2). But most of all ATP regeneration capacity within mitochondria of leukocytes was characterized by a significant $(p<0.01)$ rise (pmol/10 $0^{6}$ cells) in the verum group when compared to placebo (table 2). Such higher values of regenration capacity after washout of sodiumazide, a specific inhibitor of the electron transport capacity at complex $\mathrm{V}$ of mitochondria, reflect a significant higher energy turnover, which is not seen in the placebo group.

\section{Discussion}

Post-exercise heart rate recovery, though a readily obtainable parameter and a powerful and independent indicator of cardiovascular fitness in sports (Dimkpa 2009), is also a powerful predictor of cardiovascular and all-cause mortality in healthy adults and in those with cardiovascular diseases. This "recovery" heart rate is a determination of how long it takes the heart rate to return to normal after stop of exercising. Heart rate recovery (HRR) is mainly thought to be due to parasympathetic reactivation and has been shown to be a remarkable element to a medical and/or physical assessment of an individual in sport activities. In this regard clinical application of 
HRR after exercise with a delayed decline of heart rate has been associated with increased risk of cardiovascular mortality, autonomic dysfunction, diabetes, endothelial dysfunction, and metabolic syndrome (Cole, Blackstone et al. 1999) (Jouven, Empana et al. 2005). Similarly, HRR is associated with some cardiovascular fitness indices such as, maximum oxygen uptake, endurance capacity and central hemodynamic variables like resting heart rate, and resting blood pressure (McDonald, Hoffman et al. 1999). Thus, our data and the research work of others (Dimkpa 2009) demonstrate that heart rate can be used as an indicator determining how hard the person is exercising, but, also how to optimize recovery of the cardiovascular system following a strenuous workout. In the present experiments heart rate recovery was characterized by a faster return to baseline values within the verum group, suggesting a clear beneficial effect. As to the mode of action it was also demonstrated that only those subjects having ingested the power drink prior to the strenuous workout session demonstrated a significant ATP regeneration capacity in mitochondria. This assumption is also supported by independent data from others who evaluated synthesis of ATP in leucocytes in male athletes and who had taken the power solution for over five weeks reflecting a significant increase in the synthesis of ATP (Kirsch M et al. unpublished observation, 2012).

The positive effect of the administered power solution on previously strained musculature is readily explained by considering the constituents of the nutraceutical solution, which have a primary target at mitochondria. This is because aside form the monosaccharide ribose, it contains two necessary ingredients to achieve optimal energy production within cellular organelles, i.e. NADH (or Q1) and the active form of Q10 or ubiquinol, two necessary constituents accelerating the ATP formation within mitochondria (Fossilien 2001). In detail, benefits of ingredients of the power solution for ATP synthesis in mitochondria can be summarized as follows:

First, the sugar ribose represents a monosaccharaide with five $\mathrm{C}$ atoms (pentose) and an aldehyde group at position $\mathrm{C} 1$. It is a natural substrate within the electrical transport chain, and necessary for regeneration and the synthesis of the energy substrate ATP in mitochondria. Since extreme workout is correlated with a decline of AMP within the cell, this sugar, which does not affect blood glucose level, is a mandatory adjunct following the depletion of ADP/AMP following strenuous exercise (Segal and J.T 1958, Zimmer and Schad 1984, Pliml, von Arnim et al. 1992).

Second, by adding Q10 or ubiquinone and especially its hydrolyzed form (i.e. ubiquinol or Q10$\mathrm{H} 2$ ), the latter being characterized by a higher solubility and uptake via the intestinal tract (Hosoe, Kitano et al. 2007), is one of the most important co-enzymes acting at complex II within the electrical transport chain of mitochondria (Shults, Bealc et al. 2004).

Third, by adding Q1 or NADH, i.e. the reduced form of NAD (nicotinamide-adenine-dinucleotide) we incorporate an active $\mathrm{H}$-donor, which can be considered the spark plug to set off the energy production at complex I at the mitochondrial level (Eaton, Middleton et al. 2002). Because it had previously been demonstrated that Q1 (aka NADH) results an increased formation of ATP in athletes (Birkmayer and Vank 1996), these data corroborate our present results in aiding the restoration of energy stores.

Adding the two B-vitamins to the energy drink was deemed essential as they act upon the mitochondrial energy production pathway as co-enzymes especially in the oxydoreduction process such as the NADH-dehydrogenase, promoting a significant step in the energy production within mitochondria. Lastly, Siberian ginseng (aka eleuthero) being a full-scale member of the ginseng family, and different to the American type (panax) ginseng, has been added to the formulation because it increases energy restoration to an area of the body resulting in an increased influx of glucose to the working musculature. This favorable effect is derived from its two active promoters, i.e. the eleutherosides B \& E, two components, which are able to boost up performance, concentration and stamina as demonstrated elsewhere (Farensworth, Kinghorn et al. 1985) (Fang, Proksch et al. 1985). In addition Siberian ginseng improves blood circulation, being helpful to athletes by regulating their metabolism and allowing oxygen levels to be utilized more effectively. Taken together, the increased blood circulation and the higher energy levels within the working cell, as demonstrated in the regeneration of ATP within leucocytes, resulted in a shortening of recovery of musculature following intense physical endurance thus accelerating recovery. This can 
be considered a potential advantage when an additional demand on muscle workout, such as in competitive sports, is needed. In this regard the significant higher blood glucose level in the immediate post exercise period in our verum group mirrors a higher energy source, a favorable side effect for replenishing the exhausted glycogen pool in the musculature, an effect which very likely is related to the two ginsenoids. Such an assumption is underlined by a previous study, which had demonstrated a more efficient glucose uptake of cells following ingestion of the two ginsenoids (Freye and Gleske 2013). As a result, faster restoration of the working musculature presents an advantage in competition sport, where the recovery after workout in the immediate phase is required, which otherwise may lead into a state of energy depletion and muscle fatigue.

Within our experimental design it may be argued that max workout was not the same in both groups thus leading to the observed benefit in recovery of heart rate. Such an argument can be considered as not applicable, since both groups had reached nearly identical blood lactate levels, a reliable indicator of similar muscle strain, exceeding the so-called oxidative level.

In conclusion, the use of a specific composition in a sport drink supports mitochondrial function and ATP regeneration in healthy young college female soccer players following exposure to max workout. This is characterized by a faster recovery in heart rate and systolic blood pressure and can be attributed to an induced higher turnover of ATP within mitochondria leading to faster restoration of a previously ATP depleted musculature. It therefore is likely to be of benefit than just using carbohydrates together with proteins in the recovery period, especially since consuming too much starch or sugar may have a direct association with poorer heart pump function (Sen, Kundu et al. 2013). This is underlined by data where the glucose metabolite glucose 6-phosphate (G6P) was identified as an "evildoer", as this molecule causes stress to the heart, thereby triggering a change in the heart muscle protein.

\section{Acknowledgements:}

The authors would like to thank the Polar ${ }^{\circledR}$ Company/Switzerland for supplying us with their heart rate monitoring devices.

The participation of all female soccer players is gratefully acknowledged. Special thanks to Varenka, Marina, Kaya, Ekena, Ladina, Sinja, Anja, Nina, Ursina and Ricci for their outstanding efforts in making this study feasible.

\section{Disclaimer:}

The authors declare to have no financial ties with the originators of the sports drink Greenspeed $\AA$, neither do they hold any stocks.

Table 1

\begin{tabular}{|l|l|}
\hline Active ingredient & Dose (mg) \\
\hline Ubiquinol or Q10H2 & 50 \\
\hline NADH or Q1 & 15 \\
\hline Passion flower & 3 \\
\hline Siberian ginseng & 15 \\
\hline Nicotinamide or vitamin B3 & 54 \\
\hline Riboflavin or vitamin B2 & 5 \\
\hline Caffeine & 15 \\
\hline Vitamin E & 30 \\
\hline Ribose, a monosaccharide & 1000 \\
\hline
\end{tabular}


Table 2

\begin{tabular}{|l|l|l|l|}
\hline Parameters & Placebo & Verum & Significance \\
\hline Age (years) & $18 \pm 1.7$ & $18 \pm 1.7$ & n.s. \\
\hline Weight $(\mathrm{kg})$ & $57 \pm 6.1$ & $57 \pm 6.1$ & n.s \\
\hline Height $(\mathrm{cm})$ & $165 \pm 5.9$ & $165 \pm 5.9$ & $\mathrm{n} . \mathrm{s}$ \\
\hline Running distance $(\mathrm{km})$ & $2.4 \pm 0.2$ & $2.4 \pm 0.19$ & n.s \\
\hline Heart rate (beats/min) & $188 \pm 8$ & $187 \pm 9$ & $\mathrm{n} . \mathrm{s}$ \\
\hline Blood sugar (mmol/L) & $5.8 \pm 0.9$ & $6.5 \pm 0.8$ & $\mathrm{p}<0.005$ \\
\hline Lactate (mmol/L) & $8.3 \pm 3.3$ & $11 \pm 3.8$ & n.s. \\
\hline $\begin{array}{l}\text { ATP regeneration capacity in } \\
\text { mitochondria } \\
\text { (pmol/10 cells) }\end{array}$ & $\begin{array}{l}216.3 \\
\pm 83.1\end{array}$ & $639.8 \pm 304.1$ & $\mathrm{p}<0.01$ \\
\hline
\end{tabular}

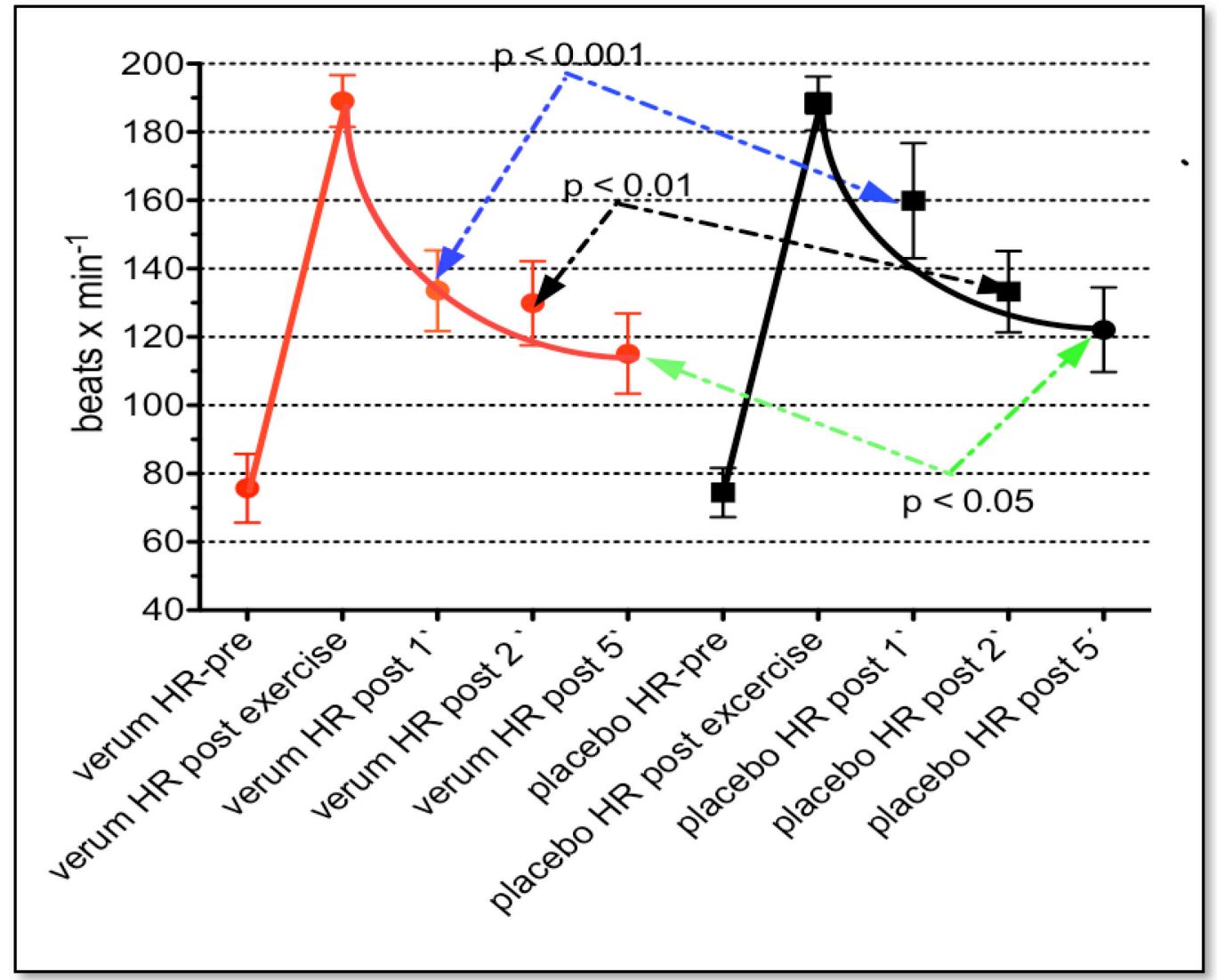

Fig. 1 


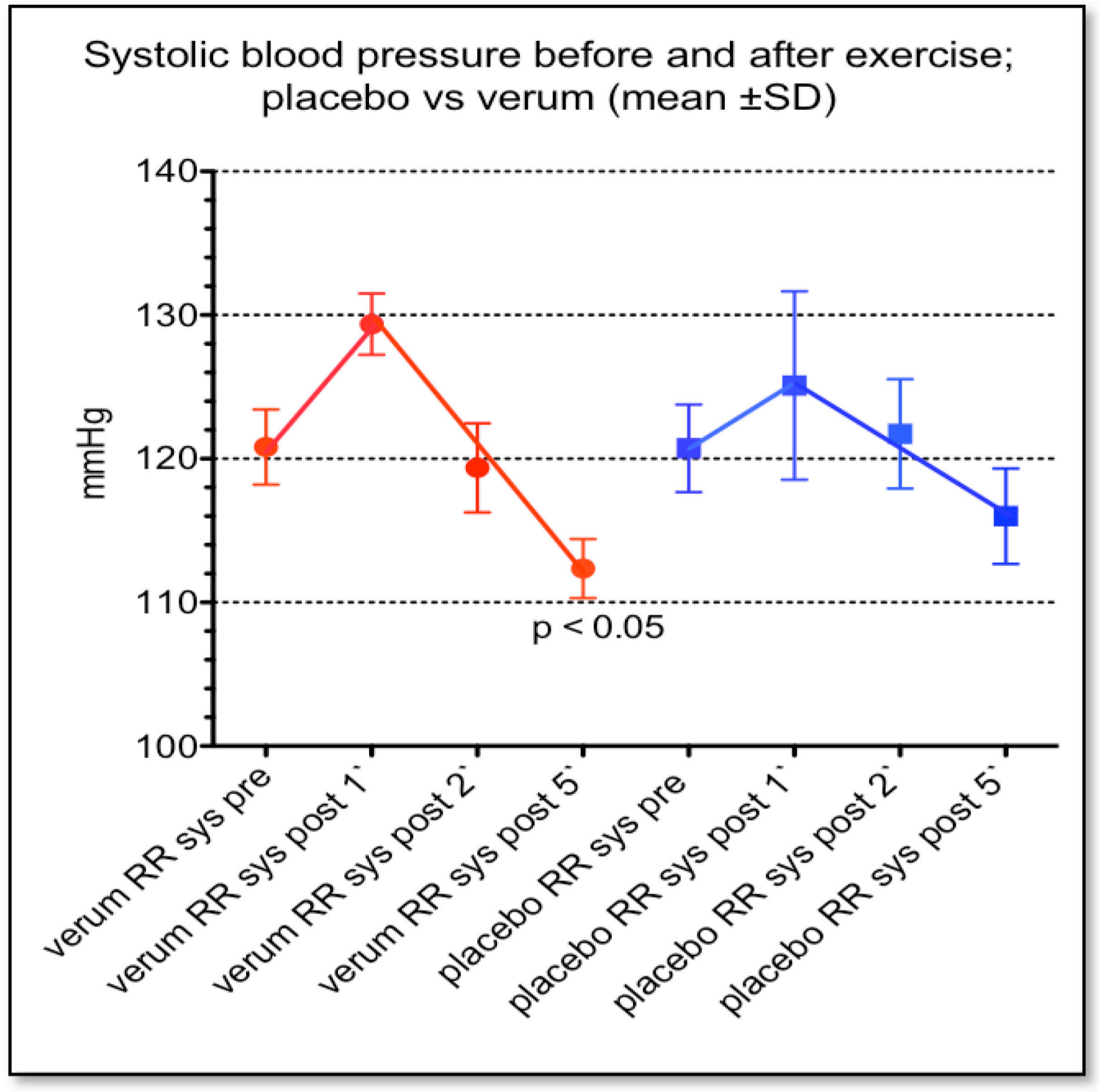

Fig. 2

\section{Legend for figures and tables:}

Table 1: Compositions of the energy drink improving energy turnover in the electrical chain reaction within mitochondria of cells

Table 2: Demographic data and significance of blood chemistry data in the placebo and the verum group following endurance running (mean $\pm \mathrm{SD}$ )

Fig. 1: Resting as well as max heart rate (HR) and heart rate recovery rate in young female soccer players in the immediate post exercise minutes with and without previous intake of a mitochondria directed nutraceutical drink (mean \pm SD)

Fig. 2: Difference in the decline of the systolic blood pressure in young female soccer players after workout, with and without the intake of a mitochondria directed nutraceutical (mean $\pm \mathrm{SD}$ ) 


\section{References}

[1] Birkmayer, J., G ,D. and P. Vank (1996). Reduced coenzyme 1 (NADH) improves pyschomotoric and physical performance in athletes. White Paper Report. New York, Menuco Corp.

[2] Bowler, M. W., M. G. Montgomery, A. G. W. Leslie and J. E. Walker (2006). "How azide inhibits ATP hydrolysis by the F-ATPases." PNAS 103: 8646-8649.

[3] Cole, C., E. H. Blackstone, F. J. Pashkow, C. E. Snader and M. S. Lauer (1999). "Heart-rate recovery immediately after exercise as a predictor of mortality." N Engl J Med 342: 13511357.

[4] Dimkpa, U. (2009). "Post-exercise heart rate recovery: an index of cardiovascular fitness." JEPonline 12: 19-22.

[5] Eaton, S., B. Middleton, H. Sherratt, M. Pourfarzam, P. Quant and K. Bartlett (2002). "Control of Mitochondrial $\beta$-Oxidation at the Levels of $[\mathrm{NAD}+] /[\mathrm{NADH}]$ and $\mathrm{CoA}$ Acylation." Adv Expert Med Biol 466: 145-154.

[6] Fang, J. N., A. Proksch and H. Wagner (1985). "Immunlogically active polysaccharides of acanthopanax senticosus." Phytochemistry 11: 2619-2622.

[7] Farensworth, N. R., A. D. Kinghorn, D. D. Soejarto and D. P. Waller (1985). Siberian ginseng (Eleutherococcus senticosus). Current state as an adaptogen. Economic and Medicinal Plant Reserach. H. Wagner, H. Hikino and N. R. Farnsworth. London, New York, Sydney, Toronto, Academic Press: 155-215.

[8] Fossilien, E. (2001). " Mitochondrial medicine--molecular pathology of defective oxidative phosphorylation." Ann Clin Lab Sci 31: 25-76.

[9] Freye, E. and J. Gleske (2013). "Siberian Ginseng Results in Beneficial Effects on Glucose Metabolism in Diabetes Type 2 Patients: A Double Blind Placebo-Controlled Study in Comparison to Panax Ginseng." Int J Clin Nutri 1: 11-17.

[10] Hosoe, K., M. Kitano, H. Kishida, H. Kubo, K. Fujii and M. Kitahara (2007). "Study on safety and bioavailability of ubiquinol (Kaneka $\mathrm{QH}^{\mathrm{TM}}$ ) after single and 4-week multiple oral administration to healthy volunteers." Regul Toxicol Pharmacol 47: 19-28.

[11] Jouven, X., J. P. Empana, S. P.J., M. Michel Desnos, D. Courbon and P. Ducimetière (2005). "Heart-rate profile during exercise as a predictor of sudden death." N Engl J Med 352: 19511958.

[12] KamMing, K. and Y. L. Hoi (2007). "Enhancement of ATP generation capacity, antioxidant activity and immunomodulatory activities by Chinese Yang and Yin tonifying herbs- A Review." Chin Med 2: 1-10.

[13] Ko, K. M., T. Y. Leon, D. H. F. Mak, P. Y. Chiu, Y. Du and M. K. T. Poon (2006). "A characteristic pharmacological action of 'Yang-invigorating' Chinese tonifying herbs: Enhancement of myocardial ATPgeneration capacity." Phytomedicine 13: 636-642.

[14] Korge, P. (1995). "Factors limiting adenosine triphosphatase function during high intensity exercise. Thermodynamic and regulatory considerations." Sports Med 20: 215-225.

[15] McDonald, T., W. E. Hoffman, R. Berkowitz, F. Cunningham and B. Cooke (1999). "Heart rate variability and plasma catecholamines in patients during opioid detoxification." $\mathrm{J}$ Neurosurg Anesthesiol 11: 195-199. 
[16] Pedersen, D. J., S. J. Lessard, V. G. Coffey, C. E.G., A. M. Wootton, T. Ng, M. J. Watt and J. A. Hawley (2008). "High rates of muscle glycogen resynthesis after exhaustive exercise when carbohydrate is coingested with caffeine." J Appl Physiol 105: 7-13.

[17] Pliml, W., T. von Arnim, A. Stablein, H. Hofmann, H.-G. Zimmer and E. Erdmann (1992). "Effects of ribose on exercise-induced ischaemia in stable coronary artery disease." Lancet 340: 507 - 510,

[18] Segal, S. and F. J.T (1958). "The metabolism of D-ribose in man." J Clinical Invest 37: 719 735 .

[19] Sen, S., B. K. Kundu, H. C.-J. Wu, S. S. Hashmi, P. Guthrie, L. W. Locke, R. J. Roy, G. P. Matherne, S. S. Berr, M. Terwelp, B. Scott, S. Carranza, O. H. Frazier, D. K. Glover, W. H. Dillmann, M. J. Gambello, M. L. Entman and T. H. (2013). "Glucose Regulation of Load-Induced mTOR Signaling and ER Stress in Mammalian Heart" J Am Heart Assoc 2(e004796 DOI: 10.1161/JAHA.113.004796).

[20] Shults, C. W., M. F. Bealc, D. Songa and D. Fontaine (2004). "Pilot trial of high dosages of coenzyme Q10 in patients with Parkinson's disease." Expt Neurol 188: 491-394.

[21] Zimmer, H.-G. and J. Schad (1984). "Ribose intervention in the cardiac pentose phosphate pathway is not species-specific." Science 223: 712 - 713. 